\title{
Impact of Social Networking Sites on Post-Partum Depression in Women: An Analysis in the Context of Bangladesh
}

\author{
Abir Saha \\ Department of Electrical and Computer Engineering \\ Indiana University-Purdue University Indianapolis, USA \\ abirsaha@iupui.edu
}

\author{
Maitraye Das \\ Department of Computer Science and Engineering \\ United International University, Dhaka, Bangladesh. \\ maitraye@cse.uiu.ac.bd
}

\begin{abstract}
Postpartum Depression (PPD) refers to moderate or severe depression in a woman after childbirth. It is strikingly common in new mothers from all regions of the world with a prevalence of around $10-15 \%$. PPD can have severe adverse effects on maternal and child health, such as suicidal tendency of the mother, infanticide as well as poor cognitive and developmental growth of the child. Despite this, few women seek medical attention due to ignorance, negligence and financial limitations; the latter is especially true for those who live in developing countries. Nowadays, social networking sites (SNS) e.g., Facebook can act as accessible and effective tools for the prevention and treatment of PPD. In this paper, we analyze the opinions and awareness level of Bangladeshi people about PPD and impact of using SNS during postpartum period on reducing PPD based on our survey $(\mathrm{N}=\mathbf{9 3})$. We also discuss possible SNSbased interventions and design implications that can effectively and feasibly reduce PPD in women in developing countries.
\end{abstract}

Keywords-Social networking sites; postpartum depression; maternal well-being; women's health

\section{INTRODUCTION}

Depression is one of the predominant factors that contribute to disability among women [1]. Women are more likely to be affected by depression compared to men (14\% of women compared to $7.3 \%$ of men [2]). While the rates of women suffering from depression during the postpartum period (i.e. the first 12 months after childbirth) are estimated to be $10-15 \%$, these rates can go as high as $20 \%$ or more in low- and mediumincome countries [3]. In several longitudinal studies conducted in Bangladesh, around $18 \%$ women during pregnancy and $31 \%$ women 6-8 months postpartum displayed depressive symptoms [36-37]. Postpartum depression (PPD) may lead to suicide, infanticide, delayed development of infant's behavioral and cognitive abilities, paternal depression, and several other negative impacts on a woman, her infant, and close ones [4]. Unfortunately, most women do not receive the necessary care and guidance to deal with their psychological issues during their first year postpartum. Even in developed countries, about half of the cases involving PPD go undetected and untreated [5]. Lack of social and familial support, society's negative attitude towards mental illness, and the notion of mood changes being a natural part of the overwhelming post-childbirth period are considered to be the main factors behind underreporting of PPD cases [6]. Lack of trained professionals, long waiting times associated with in-person psychotherapy, financial limitations, concerns about privacy, and lack of knowledge regarding depression symptoms further discourage women from seeking help [6]. Women in developing and under- developed countries in particular face more difficult situations in this regard. Therefore, there is a growing need for innovative, accessible and affordable interventions to aid postnatal psychological symptoms for women.

One way that new mothers may receive support to navigate and deal with their new responsibilities and manage physical and mental health related conditions is through support offered via Social Networking Sites (SNS) such as Facebook [7]. As of June 2017, Facebook has 1.32 billion daily active users on average and 2.01 billion monthly active users worldwide [8]. A study across 8 countries suggested that new parents use Facebook 1.3 times more often than non-parents, while new American moms post 2.5 times more status updates, 3.5 times more photos and 4.2 times more videos than nonparents [9]. Facebook has rolled out several features to cater to this new and growing demographic, such as the ability to add 'expecting a baby' as a life event and to add babies and even fetuses to a 'Friends \& Family' profile field [10]. A third-party Facebook application called 'unbaby.me' allows users to remove from their Facebook news feed what they consider to be an undesired stream of baby photos [11]. Through ethnographic studies, Gibson and Hanson [12] found that new mothers considered Facebook to be a valuable platform to maintain social connection during the postpartum period, to create a new identity, and to search for information and reassurance needed to justify their choices and actions in raising a newborn child.

Not to be limited to developed countries only, usage of Facebook and its influence on people's lives are likely to see continued growth in developing countries also. Facebook has become so pervasive in developing countries that for a large number of people, Facebook has become synonymous with internet [13]. According to Bangladesh Telecommunication Regulatory Commission, $80 \%$ internet users of Bangladesh use Facebook. Based on BTRC's statistics as of June 2017, there are approximately 74 million Internet users $(44.5 \%$ penetration rate), and 21 million Facebook users (12.7\% penetration rate) in Bangladesh, and Bangladesh is likely to see a continued growth in usage and influence of Facebook in upcoming days [14]. Due to this increasing usage statistics, Facebook periodically devises and executes new plans to facilitate internet availability in developing countries [15].

In this paper, we focus on the mothers in developing countries to figure out how social support navigated through SNS can help them to deal with postpartum depression. Our main contributions of this work are as follows: 
- We conducted an online survey with 93 participants to gain insight on the potential changes motherhood can bring about in SNS usage by Bangladeshi women.

- We statistically analyzed the data to formulate the effects of using SNS on PPD in Bangladeshi women.

- Through the survey, we shed light on the perception and awareness level of general people in Bangladesh irrespective of gender regarding PPD.

- We discussed possible design guidelines for tailoring SNS (or apps within SNS ecosystems) to provide better support to new mothers facing PPD, especially those who live in developing countries, and to satisfy their different needs such as including informationseeking, group activity for stress-relief, and privacy of sensitive information about one's mental status.

\section{RELATED WORK}

Given Internet's tremendous potential to be a viable tool in reducing depression among women during postpartum period, researchers have developed and tested several internet-based approaches to cater to pregnant women and new mothers [16]. As described in [17], MumMoodBusters showed success in reducing symptoms of depression in a sample group of postpartum women from Australia and United States. Postpartum women who underwent a randomized 11-session behavioral activation program aided by the NetMums website exhibited a lower depression score based on the Edinburgh Postnatal Depression Scale (EPDS) [18] compared to women who were in control condition (43.8\% vs 63\%) [19]. These studies show promise that online based interventions can help postpartum women suffering from depression.

A number of studies have been conducted on how mothers use different social aspects of internet, such as blogging, motherhood and parenting forums, and Facebook. The use of social media by mothers is best illustrated by the trend nicknamed 'Mommy Blogging', where mothers post public blogs and photos reflecting their children's daily lives, give advice to fellow mothers and advertise products related to babies and parenthood [20]. Based on a study conducted by Scarborough Research, 14\% mothers in United States consider themselves 'Mommy Bloggers' [21]. According to the findings of McDaniel et al. [22], blogging frequency of new mothers was correlated to the feelings of maternal well-being, support, and social connection. De Choudhury et al. [23-25] studied Facebook and Twitter use of new mothers to detect and predict sudden and extreme postpartum behavior changes. In [26], Morris studied patterns of social networking practices of new mothers, such as frequency and types of child-relate contents shared by the mothers on social media.

Another popular form of online service used by mothers to get and share parenting advice is motherhood forum, including forums hosted on parenting sites like 'babycenter.com' [27] or anonymous forums such as YouBeMom [28]. The upsides of online support groups include freedom of expression, anonymity, control over timing and extent of help one wishes to get, networking, and increased self-efficacy [29]. People feel safe to share stigmatized health related problems on online support groups due to their anonymous nature, and therefore such groups promote discussions on topics otherwise perceived to be negative [29]. In [30], a systematic review of internet-based interventions found that members of online support groups appreciated the prompt communication between members and 'anytime and anywhere' access to care.

Mixed opinions exist among researchers when it comes to internet's effects on mothers. Some think that the information exchange and online communities facilitates women empowerment [31]. However, others claim that internet only affirms norms of consumerism and femininity, and has a negative impact on the stress level, confidence, and the overall transition from pregnancy to motherhood [22]. According to some studies, excessive use of internet leads to less time spent with near and dear ones and may result in an increased loneliness, while other studies show no displacement of time with others [22]. Given these divided opinions in this regard, it is important to study the specific effects of SNS usage on women in their postpartum stage.

Most of the existing studies are conducted in developed countries where majority of the population have greater access to healthcare compared to the people of developing countries. In developing countries, resources are scarcer for the provision of mental health services and the need for such care far outweighs what local providers can offer to those who are suffering or at risk for PPD. In this paper, we target Bangladeshi people as representatives of people from developing countries to analyze the impacts of using SNS on postpartum depression in women. Additionally, we attempt to figure out the knowledge level of general people (irrespective of gender) regarding postpartum depression and their perception towards its effects.

\section{DATA COLLECTION}

We conducted an anonymous online survey to collect information about PPD experiences of new mothers and knowledge level of general people about PPD [32]. The survey was publicized through two posts in two separate closed Facebook groups with the permission of corresponding group administrators. The survey website was active throughout August 2017. We posted in Facebook groups instead of sending survey invitations to personal accounts or mailing lists to collect data from diverse population.

The survey questions and options were available in both Bengali and English. We collected demographic information, data related to SNS usage (types of SNS used, devices used, common activities, frequency of usage, privacy concerns etc.) and participants' opinions about PPD and effects of SNS usage on PPD. For mothers, we included a separate section that asked questions according to EPDS [18] about their mental state to detect whether they faced postpartum depression and their SNS usage after childbirth. The EPDS consists of 10 questions. A woman scoring 9 or more points out of maximum points 30 or indicating any suicidal ideation - that is, she scores 1 or higher on the $10^{\text {th }}$ question is considered to be moderately severe to severely depressed. Most of the questions were structured checkboxes or multiple-choice questions. Note that in case of 
multiple choice questions, summation of the percentage of different categories may exceed 100. The survey also included an optional open-ended textbox where participants could freely express their opinions, concerns, or suggestions about SNSbased interventions for reducing PPD.

\section{FINDINGS}

We received voluntary responses from 93 participants. Among them 83 were female $(89.2 \%)$ and 10 were male $(10.8 \%)$. We included male participants to collect their opinion about PPD. The mothers were asked whether they felt depressed within up to one year after childbirth. Among 70 mothers, 62 answered positively and 3 answered negatively to this question, whether another 5 were not sure. Additionally, they were asked questions according to EPDS [18] for screening of PPD. Combining the results of self-report and EPDS score, 53 mothers are considered to have moderate-tosevere PPD and 17 are without PPD. Demographic information of the participants are summarized in Table I. We do not see any significant differences between the group of mothers with PPD and the group without PPD on the basis of demographics.

\begin{tabular}{|c|c|c|c|c|}
\hline \multicolumn{5}{|c|}{ DEMOGRAPHIC INFORMATION } \\
\hline $\begin{array}{l}\text { Characte } \\
\text { ristics }\end{array}$ & Subcategories & $\begin{array}{c}\text { n (\%), } \\
\mathrm{N}=\mathbf{9 3}^{\mathrm{a}}\end{array}$ & $\begin{array}{c}\text { Mothers } \\
\text { without PPD } \\
\text { n (\%) } \\
\mathbf{N}=17^{\mathrm{a}}\end{array}$ & $\begin{array}{c}\text { Mothers } \\
\text { with PPD } \\
\text { n (\%) } \\
\mathbf{N}=\mathbf{5 3}^{\mathbf{a}}\end{array}$ \\
\hline \multirow{3}{*}{$\begin{array}{l}\text { Age } \\
\text { range }\end{array}$} & $18-25$ & $10(10.8 \%)$ & $0(0.0 \%)$ & $1(1.9 \%)$ \\
\hline & $26-35$ & $72(77.4 \%)$ & $16(94.1 \%)$ & $43(81.1 \%)$ \\
\hline & $36-45$ & $11(11.8 \%)$ & $1(5.9 \%)$ & $9(17.0 \%)$ \\
\hline \multirow{3}{*}{$\begin{array}{l}\text { Relations } \\
\text { hip status }\end{array}$} & Single & $11(12.2 \%)$ & $0(0.0 \%)$ & $0(0.0 \%)$ \\
\hline & Married & $77(85.6 \%)$ & $17(100 \%)$ & $51(96.2 \%)$ \\
\hline & Divorced & $2(2.2 \%)$ & $0(0.0 \%)$ & $2(3.8 \%)$ \\
\hline \multirow{3}{*}{$\begin{array}{l}\text { Number } \\
\text { of } \\
\text { children }\end{array}$} & 0 & $23(24.7 \%)$ & $\mathrm{N} / \mathrm{A}$ & $\mathrm{N} / \mathrm{A}$ \\
\hline & 1 & $49(52.7 \%)$ & $11(64.7 \%)$ & $38(71.7 \%)$ \\
\hline & 2 or more & $21(22.6 \%)$ & $6(35.3 \%)$ & $15(28.3 \%)$ \\
\hline \multirow{5}{*}{$\begin{array}{l}\text { Employm } \\
\text { ent status }\end{array}$} & Student & $15(16.1 \%)$ & $4(23.5 \%)$ & $3(5.7 \%)$ \\
\hline & Serviceholder & $44(47.3 \%)$ & $7(41.2 \%)$ & $28(52.8 \%)$ \\
\hline & Freelancer & $4(4.3 \%)$ & $0(0.0 \%)$ & $2(3.8 \%)$ \\
\hline & Home-maker & $16(17.2 \%)$ & $4(23.5 \%)$ & $11(20.8 \%)$ \\
\hline & Unemployed & $14(15.1 \%)$ & $2(11.8 \%)$ & $9(16.9 \%)$ \\
\hline \multirow{2}{*}{$\begin{array}{l}\text { Country } \\
\text { of living }\end{array}$} & Bangladesh & $60(69.8 \%)$ & $11(68.8 \%)$ & $30(66.7 \%)$ \\
\hline & Others $^{\mathrm{b}}$ & $26(30.2 \%)$ & $5(31.2 \%)$ & $15(33.3 \%)$ \\
\hline \multirow{4}{*}{$\begin{array}{c}\text { Highest } \\
\text { education } \\
\text { level }\end{array}$} & High school & $2(2.2 \%)$ & $0(0.0 \%)$ & $0(0.0 \%)$ \\
\hline & Bachelor's & $33(35.5 \%)$ & $4(23.5 \%)$ & $16(30.2 \%)$ \\
\hline & Master's & $55(59.1 \%)$ & $13(76.5 \%)$ & $34(64.2 \%)$ \\
\hline & $\mathrm{PhD}$ & $3(3.2 \%)$ & $0(0.0 \%)$ & $3(5.6 \%)$ \\
\hline
\end{tabular}

a. The number of participants in demographic categories does not add up to total participants as not all participants completed the demographic questions.

Others include developed countries like USA, UK, Australia, Canada, Germany, France, Singapore, South Korea etc.

\section{A. SNS Usage}

Facebook is the most popular SNS among the participants. Out of 92 responses, $90(97.8 \%)$ stated that they used Facebook. The other popular SNSs include WhatsApp (21.7\%), LinkedIn (20.7\%), Instagram (19.6\%), Google+ (12\%) etc. Out of 93 participants, 81 (87.1\%) visit SNSs multiple times a day and $12(12.9 \%)$ visit at least once a day. Mobile is mostly used to access SNS (100\%). Participants also use Laptop (20.4\%), Desktop (15.1\%) and Tablet (8.6\%).

Reading others' posts and comments and personal messaging are two common activities in SNSs like Facebook. Out of 87 responses, $65(74.7 \%)$ persons said that they mostly go through others' posts and comments and 57 (65.5\%) persons mostly do personal messaging. Other most common activities include giving likes/reactions (54\%), uploading photos/ videos $(41.4 \%)$, commenting $(33.3 \%)$, posting on own timeline $(27.6 \%)$, sharing others' posts $(19.5 \%)$, posting in different groups $(12.6 \%)$ etc. We can see that in comparison to active users (users who actively post), the number of lurkers (users who only read others' messages, but do not post themselves) are significantly higher. This user behavior is also reflected in online forums [33].

Privacy is a big concern in case of sharing personal information related health conditions in SNSs. Out of 73 responses to the question regarding sharing health information, 33 persons $(45.2 \%)$ indicated that they do not want to disclose their identity in any situation, 27 persons (37\%) expressed their willingness to disclose identity in a closed support group among people with similar conditions, whereas only 13 persons $(17.8 \%)$ said that they are not concerned about anonymity. In response to a question regarding what types of activities the user does to maintain anonymity in case of sharing or looking for answers to a personal concern, $32(65.3 \%)$ out of 49 respondents said that they search through others' posts and comments, $17(34.7 \%)$ use fake/ anonymous accounts to post the question, $13(26.5 \%)$ post the questions themselves pretending that they are asking for someone else or for no particular reason, while another $5(10.2 \%)$ ask close friends or family members to post on their behalf.

The participants were also asked what types of SNS community/ platform they prefer in case of sharing any general concern. Among 90 respondents, 61 (67.7\%) prefer personal homepage, while closed/ secret group and public group/ pages are preferred by $24(26.6 \%)$ and $5(5.6 \%)$ persons respectively. As opposed to general concerns, when participants were asked which community/ platform they prefer to share physical/ mental health related concerns, 55 out of 84 respondents (65.5\%) chose closed/secret group and 21 (25\%) chose personal message. Only $4(4.8 \%)$ persons selected public page/group while another $4(4.8 \%)$ chose personal homepage. 4 respondents said that they share health concerns face-to-face with their therapists only instead of using SNS. However, all of them live in developed countries outside Bangladesh. Among the people who preferred closed groups for sharing health concerns, 30 chose group with particular audience (e.g. female only), 23 chose support group consisting of people with similar conditions, and 18 chose group containing only close friends or family members. Only 8 persons selected closed group containing medical practitioners or psychotherapists and 6 of them live in developed countries. Considering the participants who responded to both set of questions regarding general and health related concerns, we found that these community preference of people (closed group/message vs. public group vs. personal homepage) for general and health related concerns are significantly different: $\chi^{2}(2,84)=71.24, p<0.00001$.

\section{B. SNS Usage after Childbirth and Its Effects on PPD}

Among 70 mothers, 53 were diagnosed with mild or severe PPD according to EPDS [18] and self-report. The mothers were asked to report the symptoms or conditions they felt after childbirth. Table II contains the symptoms and the percentage of mothers showing that particular symptom. 


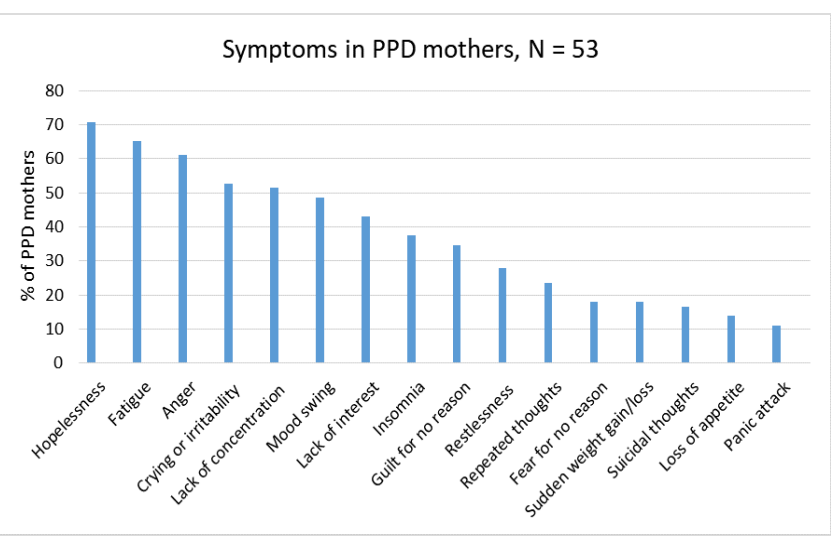

Fig 1. Percentage of PPD mothers showing different symptoms

Mothers with PPD were asked what measures they took to reduce their depression. Among 54 respondents, 22 (40.7\%) shared problems with close friends or family members, 21 (38.9\%) searched on internet for solutions, 9 (16.6\%) practiced relaxation and stress relief techniques like deep breathing, yoga, meditation, rhythmic exercises, listening to music, art therapy etc., $5(9.2 \%)$ took anti-depressant medicine, 4 took (7.4\%) help from professional therapists, 3 (5.5\%) shared thoughts on SNS, and $2(3.7 \%)$ shared experiences within an offline support group, while 20 (37\%) persons did not take any steps to reduce PPD.

After childbirth, 42 (62.7\%) among 67 respondent mothers used SNS multiples times a day, $19(28.4 \%)$ used at least once a day, $3(4.5 \%)$ used at least once a week and another $3(4.5 \%)$ used at least once a month. On the contrary, in general, among these 67 mothers, $58(89.6 \%)$ use SNS multiple times a day and $9(13.4 \%)$ use at least once a day. This decline in SNS usage after childbirth is significant $\left(\chi^{2}(1,67)=10.0894, p=\right.$ $0.0015)$. However, SNS usage rates do not vary significantly among mothers with or without PPD. Among 53 PPD mothers, $34(64.2 \%)$ used SNS multiples times a day after childbirth, 15 $(28.3 \%)$ used at least once a day, $3(5.6 \%)$ used at least once a week and $1(1.9 \%)$ used at least once a month. On the other hand, 8 (57.1\%) among 14 respondents without PPD used SNS multiple times a day after childbirth, $4(28.6 \%)$ used at least once a day, and $2(14.3 \%)$ used at least once a month. Test result is $\left(\chi^{2}(2,67)=0.6486, p=0.723\right)$.

As a follow up question to this, we asked what types of activities mothers mostly did within SNS after childbirth. Among 53 respondents, 32 (60.4\%) shared photos of child, 11 $(20.8 \%)$ added life events with the child, and 18 (34\%) asked questions regarding child's health and developmental growth. As opposed to these child-related queries and updates, only 7 $(13.2 \%)$ mothers asked questions about their physical conditions, and $5(9.4 \%)$ shared concerns about their mental health and depression. Besides actively posting concerns, 29 $(54.7 \%)$ mothers searched through others' posts and comments to find answers to their queries. Among 68 respondents, 23 (33.8\%) mothers felt that their depression decreased using SNS and $34(50 \%)$ did not feel any effect, while $11(16.2 \%)$ felt that their depression increased. Among 34 mothers who actively posted their depression related concerns and/or searched through others' posts for solution, $21(61.8 \%)$ felt that their depression decreased, $4(11.8 \%)$ felt that their depression increased, while $9(26.4 \%)$ did not feel any change. As opposed to these, among 20 mothers who only posted childrelated updates but did not share any health concerns, $5(25 \%)$ felt that their depression decreased, $5(25 \%)$ felt that their depression increased and the rest $10(10 \%)$ mothers did not feel any effect of using SNS on their depression. Thus, finding solutions to health concerns and sharing experiences within SNS by actively posting and/or searching through others' post reduces depression in mothers significantly compared to activities that include sharing child-related updates only $\left(\chi^{2}(2\right.$, 54) $=6.84, p=0.0327<0.05)$.

To better understand the effects of using SNS on PPD, we asked the mothers about the probable reasons behind any increase or decrease in depression due to using SNS. We received 49 responses to the question related to the causes of increased depression. The causes include seeing others' happy stories (63.2\% votes), physical stress due to excessive usage of SNS, e.g., headache, sore eye etc. (28.6\% votes), news of violence or stressful events (26.5\% votes), guilt for wasting too much time over SNS (26.5\% votes), and obsession with SNS (20.4\% vote). One mother wrote:

"It seemed that everyone was moving on with their lives whereas I was stuck at home breastfeeding a newborn. I am a workaholic who enjoys her work very much. I love my babies, but I don't like being at home 24/7."

39 mothers voted for the causes of their depression decreasing due to using SNS. These causes include the realization that others can also face similar conditions $(51.3 \%$ votes), sharing experience with other PPD mothers $(41 \%$ votes), getting suggestions from fellow mothers (43.6\% votes) and medical practitioners/ therapists $(12.8 \%$ votes $)$, and receiving appreciations and complements from others (41\% votes). One participant commented:

"I had little support from family and friends after losing a child after birth. The online groups with mothers who lost a child helped me a lot. Only a mother who has lost a child knows how to talk to another childless mother. So, I appreciate the support from these mothers all over the world to help me see the light at the end of my darkest times."

\section{Opinions regarding PPD and SNS-based Interventions}

This section of our questionnaire was available to all the participants irrespective of gender or marital status. The participants were asked about their general thoughts regarding women feeling depressed after childbirth. Among 68 responses to this question, $60(88.2 \%)$ acknowledged that PPD is a common problem that needs proper measures. Only $8(11.8 \%)$ respondents opined that PPD is just a 'phase' which goes away naturally without any outside help. 6 of these 8 persons were either male or female who never faced PPD.

Out of 90 respondents, $82(91.1 \%)$ were familiar with the term PPD or its synonyms, e.g., baby blue, perinatal depression, postnatal depression etc. Only 6 persons $(6.7 \%)$ never heard of any such terms and $2(2.2 \%)$ persons were not sure. 85 persons responded to the follow up question; from which source they had first leant about PPD. Among different sources, SNS platforms like Facebook received the most votes 
(43.5\%) followed by Internet search engines e.g., Google, $(24.7 \%)$, health related books $(12.9 \%)$, doctors/therapists $(10.6 \%)$, and family/friends $(8.2 \%)$.

Among 83 respondents, 55 (66.3\%) and 19 (22.9\%) respectively thought that SNS-based interventions can be or might be helpful in reducing PPD. Only 9 persons $(10.8 \%)$ thought that SNS-based interventions cannot have any positive effect in reducing PPD. In response to the question about what type of SNS-based interventions can possibly help in reducing PPD, $47(58.7 \%)$ out of 80 respondents voted for events, activities or trends to increase awareness about PPD. Other popular measures include closed support group consisting of people with similar conditions $(55 \%)$, online communication with therapists and medical practitioners $(50 \%)$, and SNSbased apps for stress-relief techniques $(27.5 \%)$. When asked as whether the participant will use or encourage others to use SNS-based support groups/ apps dealing with PPD reduction, 53 out of 83 respondents came up with a positive answer, 19 with a somewhat positive answer and 11 with a negative answer. Among the persons who answered negatively, 3 were male and 7 lived outside Bangladesh in developed countries. Some of them emphasized that women should seek help from doctors/therapists directly instead of relying on SNS. One participant living in USA wrote:

"My insurance had free support for new moms. Every week a caregiver called me and talked to me regarding my diet, health and all. That helped a lot. Not necessarily I need to reach out to unknown caregivers in social media if you have more trustworthy professional help from your doctor's office."

\section{Another participant living in Australia commented:}

"The doctors should inform would-be parents about such depression so that mothers won't wonder about what's wrong with them. They can be prepared knowing they might be affected and they may need professional help."

\section{DESIGN IMPLICATIONS}

These insights into Bangladeshi mothers' SNS use patterns suggest design and usability changes that such platforms might consider to optimally serve this demographic. For example, large portion of PPD mothers' tendency to get depressed watching others' happy stories and stressful events/violence could be explicitly handled by SNS like Facebook. De Choudhury et al. [23-25] have observed, social media traces can be used to detect warning signs of PPD. Facebook can intelligently tailor homepages of high risk PPD mothers so that less stressful or unsettling events are visible to them.

Large number of participants expressed that they feel relieved and relaxed by sharing their experiences with other mothers who also face similar conditions. Moreover, therapists and medical practitioners can remotely serve greater population, if they maintain more online presence. As number of professional therapists and affordability of general people are low in developing countries, remote health service leveraged through SNS-based closed support groups containing therapists and doctors can be considered as a feasible measure. However, most of the users do not want to ask explicitly about sensitive questions even within a closed group of limited audience. To maintain anonymity, people mostly search through others' posts and often use fake ID, take help from close friends or family, and sometimes even act pretentious while posting questions related to sensitive health concerns. SNS like Facebook can handle these fake IDs and pretentious attempts by providing designated anonymous accounts used by the members of closed/secret groups. One particular women only group called "Meye: A Sisterhood" maintains an account dedicated to anonymous stories, which gives the users an opportunity to seek help without revealing their identity [34]. This account is controlled by the admins of the corresponding group and passed over to members through email, when someone wants to share their stories anonymously. However, this creates a heavy burden on the admins and consequently, cannot provide immediate help or support to the user, when needed. Moreover, as email address is used to pass over the anonymous account's credentials to a member, the member's identity might be revealed through her email. This might force the member to use a fake email that does not contain her name or identifying information. Besides, admins have greater control over the procedure and that can also hamper privacy of the users. If such a feature of anonymous account within each secret/ closed group is added and controlled by Facebook itself, it can work as a platform for women to share their concerns easily without the fear of being identified and thus, stigmatized.

Self-help techniques such as yoga, meditation, exercise, music therapy and art therapy are often used by PPD mothers for relaxation and stress relief. However, maintaining continued practice of these techniques individually is difficult without outside motivation. While several stand-alone websites have been developed for these techniques, an SNS-based intervention delivered through Facebook may offer additional advantages. Facebook users visit the site very frequently, which increases their potential exposure to any intervention provided through this site and helps to overcome retention and increase adherence to challenges. Moreover, presenting these practices in the form of a group activity or challenge within one's peer network can develop engagement and interest among the mothers. For example, we often see challenges and group activities being trendy over Facebook [35].

For new mothers and pregnant women, social networking creates a sense of being connected, because it keeps them connected to their friends and family without the need of leaving home. Sharing personal success stories on such sites reinforces perception of social support. Mothers also experience vicarious learning by reading about the experiences of other mothers. Feedbacks from SNS reduce mothers' stress, marital conflict, and depressive symptoms, and thereby facilitate positive feelings and experiences [22]. SNS can also be a useful tool in increasing social awareness among general people as well as one's family and friends. Timely support and empathy from husband and other family members are inevitable for a PPD mother to recover quickly. Events and trends can be effective in making family members aware of their responsibilities towards mothers who are in risk of PPD.

\section{A. Limitations}

While our study offers systematic insights into effects of SNS use on PPD in the context of Bangladesh, limitations of our study suggest valuable avenues for future work. Our 
survey population is not balanced in terms of gender. To find more rigorous idea about general people's opinion regarding PPD, responses from more male participants should be collected. Our study is based on a survey conducted online. Ethnographic interviews need to be conducted to validate our findings from the survey and also to gather more subjective views. Moreover, all the participants of our survey have completed at least high school education. Thus, our study does not include feedbacks from illiterate or marginally literate population. Large number of people from this population have access to internet and regularly use SNS like Facebook. Incorporating their feedback can significantly change findings from our survey. Also, our study did not examine the intersection of fatherhood and SNS use. Understanding differences in the way SNS use can affect fathers and mothers may be a valuable area of inquiry. Additionally, our study can be extended to find design implications for helping mothers with affective disorders during prenatal and pregnancy period.

\section{CONCLUSION}

In this paper, we have discussed the findings of a survey we conducted on postpartum depression (PPD) and its relation to the activity of women on social networking sites (SNS) during their postpartum period. We statistically analyzed our survey results to determine how social networking impacts several aspects of the depression phase, the factors that reduce or increase depressive symptoms in women, and the measures women take to find solutions to these symptoms. Based on the answers given by survey participants, we suggested a set of design guidelines SNS and/or associated applications may adopt to help postpartum women feel more secure and confident while seeking advice related to their postpartum mental health problems within SNS ecosystems.

\section{REFERENCES}

[1] A. J. Ferrari et al., "Burden of depressive disorders by country, sex, age, and year: findings from global burden of disease study 2010," PLoS Med. 10 (11), e1001547, 2013.

[2] Z. Steel et al., "The global prevalence of common mental disorders: a systematic review and meta-analysis 1980 -2013," International Journal Epidemiol. 43 (2), pp. 476 -493, 2014.

[3] M.W. O'Hara, A. M. Swain, "Rates and risk of postpartum depression — a meta-analysis," Int. Rev. Psychiatry 8 (1), pp. 37 -54, 1996.

[4] S. M. Haga et al., "A longitudinal study of postpartum depressive symptoms: Multilevel growth curve analyses of emotion regulation strategies, breastfeeding self-efficacy, and social support," Archives of Women's Mental Health, 15, pp. 175-184, 2012.

[5] http://www.who.int/mental health/management/depression/wfmh paper depression_wmhd 2012.pdf

[6] C. Dennis, and L. Chung-Lee, "Postpartum depression help-seeking barriers and maternal treatment preferences: A qualitative systematic review," Birth, 33(4), pp. 323-331, 2006.

[7] M. E. Morris, S. Consolvo, S. Munson, K. Patrick, J Tsai, A. D. I. Kramer, "Facebook for health: opportunities and challenges for driving behavior change," in Proceedings of CHI' 2011, Canada, pp. 443-446.

[8] https://newsroom.fb.com/company-info/

[9] https://www.facebook.com/iq/articles/meet-theparents?ref=-wpinsights $\mathrm{rd}$
[10] J. Van Grove, "Facebook lets expectant parents add unborn children to friends \& family," Mashable, August 1, 2011.

[11] A. Considine, "Making facebook less infantile," The New York Times. August 8, 2012.

[12] L. Gibson, and V. L. Hanson, "Digital motherhood: how does technology support new mothers?," in Proceedings of CHI 2013.

[13] http://www.fudzilla.com/news/36984-developing-countries-thinkfacebook-is-the-internet

[14] http://www.internetworldstats.com/asia.htm\#bd

[15] https://www.forbes.com/sites/kathleenchaykowski/2016/04/14/facebook -launches-two-new-wireless-internet-systems-to-improve-access-indeveloping-urban-areas/\#6644f811f13b

[16] E. W. Lee, F. C. Denison, K. Hor, and R. M. Reynolds, "Web-based interventions for prevention and treatment of perinatal mood disorders: a systematic review," BMC Pregnancy and Childbirth, 16:38, 2016.

[17] B. G. Danaher et al., "MomMoodBooster: Web-based intervention for postpartum depression. Feasibility trial results." Journal Med. Internet Res. 15 (11), e242, 2013.

[18] https://psychology-tools.com/epds/

[19] H. A. O'Mahen et al., "Internet-based behavioral activation-treatment for postnatal depression (NetMums): a randomized controlled trial," Journal Affect. Disord. 150, pp. 814-822, 2013.

[20] J. H. Mendelsohn, “Don't bother mommy. I'm too busy building my brand," The New York Times, March 12, 2010.

[21] S. Laird, "The rise of the mommy blogger," Mashable. May 8, 2012.

[22] B. T. McDaniel et al., "New mothers and media use: associations between blogging, social networking, and maternal well-being," Maternal and Child Health Journal, 16(7), pp 1509-17, 2012.

[23] M. De Choudhury, S. Counts, and E. Horvitz, "Major life changes and behavioral markers in social media: case of childbirth," in Proceedings of CSCW 2013.

[24] M. De Choudhury, S. Counts, and E. Horvitz, "Predicting postpartum changes in emotion and behavior via social media," in Proceedings of CHI 2013.

[25] M. De Choudhury, S. Counts, E. Horvitz, and A. Hoff A, "Characterizing and predicting postpartum depression from shared facebook data," in Proceedings of CSCW 2014.

[26] M. R. Morris, "Social networking site use by mothers of young children," in Proceedings of CSCW 2014.

[27] https://www.babycenter.com/

[28] S. Y. Schoenebeck, "The secret life of online moms: Anonymity and disinhibition on YouBeMom.com," in Proceedings of ICWSM 2013.

[29] M. Evans, L. Donelle, and L. Hume-Loveland, "Social support and online postpartum depression discussion groups: A content analysis," Patient Education and Counseling, 87, pp. 405-410, 2012.

[30] F. Griffiths et al., "Why are health care interventions delivered over the internet? A systematic review of the published literature," Journal Med Internet Res, 8, e10, 2006.

[31] W. Hall and V. Irvine, "E-communication among mothers of infants and toddlers in a community-based cohort: A content analysis," Journal of Advanced Nursing, 65, 175-183.

[32] https://goo.gl/forms/7rW2Xi86SYNpFNaE2

[33] T. van Mierlo, "The $1 \%$ rule in four digital health social networks: an observational study," J. Med Internet Res, 16(2):e33, 2014.

[34] https://www.facebook.com/meyenetwork/

[35] http://www.goodnet.org/articles/4-facebook-challenges-that-flood-yournews-feed-good-vibes-list

[36] H. E. Nasreen, M. Edhborg, M. Petzold, Y. Forsell, and Z. N. Kabir, "Incidence and risk factor of postpartum depressive symptoms in women: A Population based prospective cohort study in a rural district in Bangladesh," Journal of Depression and Anxiety, 4, 180, 2015.

[37] A. Williams et al., "Cultural attitudes toward postpartum depression in Dhaka, Bangladesh,” Journal of Medical Anthropology, 2017. 